\title{
Meta
}

Journal des traducteurs

Translators' Journal

\section{Lederer, Marianne (1994) : La traduction aujourd'hui. Le modèle interprétatif, Paris, Hachette, " collection F ", 224 p.}

\section{Paul A. Horguelin}

Volume 40, numéro 4, décembre 1995

URI : https://id.erudit.org/iderudit/003121ar

DOI : https://doi.org/10.7202/003121ar

Aller au sommaire du numéro

Éditeur(s)

Les Presses de l'Université de Montréal

ISSN

0026-0452 (imprimé)

1492-1421 (numérique)

Découvrir la revue

Citer ce compte rendu

Horguelin, P. A. (1995). Compte rendu de [Lederer, Marianne (1994) : La

traduction aujourd'hui. Le modèle interprétatif, Paris, Hachette, « collection F ",

224 p.] Meta, 40(4), 659-663. https://doi.org/10.7202/003121ar

Ce document est protégé par la loi sur le droit d'auteur. L'utilisation des services d'Érudit (y compris la reproduction) est assujettie à sa politique d'utilisation que vous pouvez consulter en ligne.

https://apropos.erudit.org/fr/usagers/politique-dutilisation/
Cet article est diffusé et préservé par Érudit.

Érudit est un consortium interuniversitaire sans but lucratif composé de l'Université de Montréal, l'Université Laval et l'Université du Québec à Montréal. Il a pour mission la promotion et la valorisation de la recherche. https://www.erudit.org/fr/ 
LEDERER. Marianne (1994): La traduction aujourd'hui. Le modele interprétatif, Paris, Hachette, «collection F», 224 p.

D'entrée de jeu, le nom de l'auteur et le sous-titre de l'ouvrage indiquent au lecteur à quelle enseigne il va loger. Marianne Lederer, rappelons-le, est directeur de l'École Supérieure d'Interprètes et de Traducteurs (ESIT), de l'Université de la Sorbonne Nouvelle. Auteur de plusieurs ouvrages et articles sur l'interprétation et la traduction, elle a participé, aux côtés de Danica Seleskovitch. à l'élaboration de la «théorie interprétative», dont le manifeste remonte au début des années 70 . Depuis, cette théorie a fait école. non sans susciter toutefois quelques réserves.

Dans un court Avant-propos, l'auteur précise son objectif, qui est de donner cune vue générale et structurée» de la thèse qu'elle défend, en espérant que son entreprise aura «des retombées sur la façon d'enseigner la traduction et par conséquent sur sa pratique». Sont aussi posés les premiers postulats : «La démarche du bon traducteur est fondamentalement la même, quelles que soient les langues et quel que soit le texte en cause. La recherche du sens et sa réexpression sont le dénominateur commun à toutes les traductions. $|\ldots|$ Un seul et même argument sous-tend cet ouvrage : tout est interprétation. La traduction ne fait pas exception. [...] Le principe interprétatif s'applique à tous les genres de textes, de la poésie au texte le plus technique." Un sommaire très détaillé annonce la structure de l'ouvrage, qui se divise en deux grandes parties d'égale longueur : I. Aspects théoriques de la traduction: II. Les pratiques de la traduction.

Dans un premier chapitre intitulé «Interpréter pour traduire», Mme Lederer définit l'acte de traduire, qui "consiste à comprendre un texte, puis, en une deuxième étape, à réexprimer ce texte dans une autre langue» ; la définition du processus de la traduction est sensiblement la même, sauf que s'y intercale une étape intermédiaire : la déverbalisation de la forme linguistique du texte. Les notions clés sont ensuite précisées : langue. parole. texte, mais principalement interpréter, sens, compréhension. "C"est volontairement que je joue sur les deux acceptions du mot «interpréter» qui renvoie à la fois a la façon dont opèrent les interprètes de conférence et à la compréhension en profondeur et à la restitution en clair d'un texte étranger»... mais le mot a aussi une "coloration tendancieuse" provenant de la crainte d'un "débordement de sens dès lors qu'il ne serait pas restreint aux significations linguistiques». Qu'est-ce donc que le sens? Citant Sartre, Piaget, Lacan et Freud, Mme Lederer nous dit que le sens n'est pas une somme de mots, c'est «un ensemble déverbalisé. retenu en association avec des connaissances extra-linguistiques». Quant à la saisie du sens, elle «n'est pas le produit d’étapes successives mais d'une seule démarche de l'esprit. On ne comprend pas un texte d'abord au niveau de la langue, puis à celui du discours, mais d'emblée au niveau du discours". "L'unité de sens, fusion en un tout du sémantisme des mots et des compléments cognitifs. n'existe qu'au plan du discours.» La compréhension d'un texte suppose "une compétence linguistique et un savoir encyclopédiquen: il faut comprendre l'explicite de la langue. mais aussi les implicites - présupposés et sous-entendus, auxquels s’ajoutent ale contexte cognitif constitué par les connaissances acquises à la lecture du texte» et "le bagage cognitif. connaissances linguistiques et extra-linguistiques emmagasinées $[\ldots \mid$ dans la mémoire».

Si «le sens est ce que veut dire un auteur», quelles sont les limites de l'interprétation de ce vouloir dire? "Le traducteur qui se ferait exégète, l'interprète qui se ferait herméneute transgresseraient les limites de leurs fonctions» (D. Seleskovitch). Plus précisément. c’est «l'analyse justificative» effectuée par le traducteur au terme du processus qui doit permettre de vérifier à la fois l'exactitude de la traduction et son adaptation au destinataire. Ici, $\mathbf{M}^{\mathrm{me}}$ Lederer cite abondamment Jean Delisle, comme elle le fait pour l'étape de la réexpression ou reverbalisation, rapidement abordée. Il ne sera plus guère 
question de la réexpression dans le reste de l'ouvrage : on peut donc en conclure que le préalable précédemment énoncé - "Seule une excellente maîtrise de la langue d'arrivée permet la réexpression adéquate du sens» - est considéré comme une garantie suffisante.

Si le chapitre ler portait principalement sur le sens - sa saisie et son interprétation - le chapitre 2 vient préciser la notion fondamentale d'équivalence. Fondamentale parce que «la traduction interprétative est une traduction par équivalences, [alors que] la traduction linguistique est une traduction par correspondances». De prime abord, on pourrait penser qu'il s'agit là de deux modes de traduction distincts : "ll existe deux approches de la traduction, qui ont l'une et l'autre leurs défenseurs», d'un côté les traducteurs «bien formés et bien informés» qui traduisent des textes, de l'autre, ceux qui recherchent systématiquement des correspondances et traduisent des langues. Cette conception manichéenne de la traduction. si elle fait le bonheur des polémistes, n'est évidemment pas conforme à la réalité. et nous en aurons la confirmation à la fin du chapitre : "Correspondances et équivalences sont intimement liées dans le processus de la traduction. Jamais les unes ne l'emportent intégralement sur les autres.» Entre-temps, des exemples viennent expliciter les notions d'équivalence, cognitive et affective. de synecdocte en traduction (ici sont cités Vinay et Darbelnet), de "génie de la langue» ou idiomaticité. Passant à la traduction par correspondances, $\mathbf{M}^{\mathrm{mc}}$ Lederer en restreint l'application à des cas très limités: "ll peut s'agir de mots choisis délibérément, d'une liste de mots ou encore de termes techniques au référent précisément cerné." Nous pensons qu'il serait facile de montrer que les correspondances possibles de mots et même de structures sont beaucoup plus fréquentes dans les «bonnes» traductions, du moins lorsqu 'il s'agit de langues germaines comme l'anglais et le français. Aux "avatars de la traduction par correspondance" (traduction mot à mot. traduction des motivations et des phrases hors contexte) succèdent les faux problèmes (mots intraduisibles, vides lexicaux. pertes et gains) «découlant d'une conception linguistique de la traduction». Le paragraphe «L'étrangeté de la langue étrangère" évoque l'opposition entre «sourciers» (Meschonnic, Berman) et "ciblistes" (Nida, Margot), selon la terminologie de J.-R. Ladmiral, et le chapitre se termine par la fausse question séculaire : fidélité ou liberté ?', à laquelle $\mathbf{M}^{\text {me }}$ Lederer donne une réponse qui fera consensus: "Le débat liberté/fidélité est tranché par la dualité équivalences/correspondances de toute traduction réussie." Si le traducteur peut et doit exercer sa liberté "par rapport à la forme de loriginal, il ne dispose pas de cette liberté par rapport au sens. Le corollaire de la liberté en traduction est la fidélité au sens. compris non pas en tant qu'esprit par opposition à la lettre. mais en tant qu'effet global du texte sur le destinataire." Qui ne souscrirait pas à ce principe, garant de la créativité du traducteur ? C'est son application pratique qui fait problème.

On a souvent reproché aux traductologues et autres théoriciens d'échafauder de belles théories, de séduisants modèles en se gardant bien de les soumettre au banc d'essai. On ne fera pas ce reproche à $M^{\text {ne }}$ Lederer : sa démonstration de la traduction par équivalences s'appuie sur deux courtes (une ou deux phrases) traductions de l'allemand, dont une technique, et un passage d'une dizaine de phrases extraites du roman de John Steinheck. Cannery Row, traduit par Magdeleine Paz (Gallimard, 1948). L'auteur précise qu'elle a choisi ces traductions publiées "parce que je ne leur ai trouvé aucun défaut". ajoutant que le chapitre XIV d'où provient l'extrait de Cannery Row" "peut être lu, et sa traduction appréciée, sans qu'il soit nécessaire de connaître l'ensemble du livre" : l'original et la traduction du chapitre sont néanmoins reproduits en annexe. $\bar{A}$ mesure qu'on avance dans la traduction, on ressent un malaise grandissant : équivalences pour le moins bizarres, omissions et rajouts non évidents, appauvrissement presque systématique du style, le tout accompagné de justifications peu convaincantes. On dérive vers un nouveau mode de traduction où sont oubliés les grands principes sur la saisie et l'interprétation du 
sens énoncés au chapitre 2, sur les limites de la déverbalisation qui «découle de l'intime compréhension du sens d'un passage de l'original» et "n'entraîne che $z$ le professionnel ni perte ni erreur d'information". Le traducteur de Cannery Row' "se représente la scène, [...] il transcende la langue pour percevoir à travers elle le vouloir dire de l'auteur. Cette vision, ce sentiment [... | l'inspirent". Hélas, on aboutit alors à une traduction qui, pour nous, loin d'être un modèle, est presque une parodie. Un tel écart d'appréciation peut évidemment surprendre. Aussi, pour convaincre le lecteur ayant une connaissance minimale de la langue et de la culture américaines que nos réserves ou interrogations ne portent pas sur des vétilles ni ne sont inspirées par quelque malveillante querelle de clocher ou d'école, voici quelques spécimens d'interprétation qui devraient l'inciter à "faire le détour» (il est naturellement conseillé de lire la mise en situation fournie à la page 54 et d'analyser les «équivalences» dans le contexte du chapitre XIV, annexe 1 ).

The corrugated iron of the canneries

The street is silent of progress and business.

dogs choosing whereon to pee

the gophers [...] drag flowers into their holes

the bouncer $[\ldots]$ the bouncer

one wore a tiny yellow straw boater

The soldier had a large brown paper bag filled with cold canned beer.

The watchman, a dark and surly man, had seen them. la ferraille des conserveries

Le progres, les affaires, tout dort.

les chiens en quête de leur provende

les raupes [...] ramènent des fleurs dans leurs trous

le costaud l...l le gérant

l'un avait le chef adomé d'une paille jaune

Le soldat portait un cabas de papier brun, rempli de bière en boîtes.

Le gardien de nuit. un c'orps sombre ef morose, les avait apersus. of son cocker: un chien sombre et morose comme lai. les arait apersus aussi.

"Comment juger l'équivalence ?" Mme Lederer énumère les cinq critères de W. Koller (Einführung in die Übersetzungswissenschaft. Heidelberg, 1992) et conclut qu'ils ont été respectés. Quelques pages plus loin, on nous présente les trois paramètres de la fidélité selon Amparo Hurtado: "Le vouloir dire de l'auteur. la langue d'arrivée. le lecteur». Les balises ne manquent donc pas, mais la question demeure : Est-il possible que John Steinbeck ait, presque à chaque phrase, incorrectement exprimé son vouloir dire ? Ou ne sommes-nous pas plutôt en présence d'un autre «testament trahi" (Kundera) ? Le lecteur jugera.

Cette zone de turbulence passée, on aborde le dernier chapitre de la première partie : "Langage et traduction". Laissons l'auteur résumer ce chapitre : «Un rapide survol des théories linguistiques de ce dernier siècle permettra de mieux situer la traductologie par rapport aux linguistiques structurale et générative et de montrer son originalité par rapport aux tendances plus récentes. J'espère ainsi faire apparaître les raisons pour lesquelles la traduction doit être traitée sur un plan autre que linguistique." Après avoir rappelé que ala traduction est objective sur le plan des idées mais subjective sur celui de la formulation», $\mathrm{M}^{\text {me }}$ Lederer conclut : "On aura vu par les exemples que $\mathrm{j}$ 'ai donnés que le bon traducteur interprète au meilleur sens du terme et, ce faisant, fait mentir l'adage "traduttore-traditore".» Ce n'est pas évident.

Pour illustrer, dans la deuxième partie, "Les problèmes pratiques de la traduction", le directeur de l'ESIT a sélectionné les deux meilleures traductions d'un texte de Art Buchwald, produites par des étudiants ayant suivi les séminaires de doctorat. Reproduites en annexe, ces traductions nous rassurent : elles sont conformes aux principes et critères précédemment énoncés et respectent en particulier le sens et le style du texte original 
(quant au respect de la langue d'arrivée. on aurait dû enseigner à ces étudiants-traducteurs que pour distinguer les interlocuteurs dans un dialogue, on utilise le tiret en français, et non les guillemets comme en anglais). Les problèmes de traduction étudiés sont : l'absence de déverbalisation (transcodage des mots et des phrases), la fidélité («Elle ne peut pour nous qu'être fídélité aux différents aspects du sens»), le transfert du culturel (condamnation de l'ethnocentrisme : "Le bon traducteur s"interdit de "naturaliser" la culture de l'original [... II doil] donner au lecteur étranger des connaissances supplémentaires. minimum mais suffisantes pour entr'ouvrir la porte qui mène à la connaissance de l'autre." Beau principe auquel on ne peut que souscrire, mais qui semble condamner la traduction de Cannery Row et ses taupes herbivores...

Les trois derniers chapitres traitent daspects particuliers de la traduction: "Traduction et enseignement des langues", "La traduction en langue étrangère» et «La traduction automatique dans la perspective de la traduction humaine». Ils justifient le titre de l'ouvrage - La traduction aujourd'hui - et présentent une bonne synthèse des questions abordées: historique et mise à jour jusqu au début des années 90. Après Ladmiral et Delisle, Marianne Lederer établit une distinction entre la traduction pédagogique et la traduction professionnelle. Malheureusement, l'exemple utilisé est à nouveau peu convaincant : il s'agit d'une phrase de Graham Greene où figure l'énoncé "she meant nothing to Philip", traduit "pédagogiquement" par "elle ne signifiait rien pour Philippe». Commentaire : "Sous la plume d'un traducteur non soumis aux contraintes d'une édition bilingue, la phrase anglaise aurait appelé des équivalences, par exemple : "Philip n'arrivait pas à la placer", ou "Philip ne savait pas quoi penser d'elle"». Belle mais infidèle interprétation. (Lorsque la première Française chef de gouvernement a déclaré : «Je n'en ai rien à cirer», son vouloir dire n'était pas «Je n'arrive pas à situer le problème»!) «Les limites de la traduction en langue étrangère» nous font connaître une intéressante thèse de doctorat (A. Rydning. Université d'Oslo, 1992) sur l'acceptabilité de la traduction, qui «dépend en grande mesure du degré de connaissance de son destinataire"; c'est une enrichissante contribution à l'explicitation du critère "Adaptation au destinataire». Le chapitre sur la traduction automatique rappelle qu'à côté de la traduction humaine, il existe maintenant trois modes faisant intervenir l'ordinateur : la TA intégrale, la TA assistée par l'homme, et la traduction humaine assistée par l'ordinateur. Après avoir passé en revue les différences et les similitudes entre les opérations de la machine et celles de l'homme. l'auteur présente les synthèses et travaux récents, notamment le système de prétraduction de Claude Bédard décrit dans Mota (37-4, 1992). En dernier lieu, on trouvera à la fin de l'ouvrage : un glossaire d'une trentaine de termes désignant "les notions développées dans le cadre de la théorie interprétative de la traduction», une bibliographie et un index des notions.

Avant de conclure, il nous faut, à regret, constater une autre déficience surprenante de La traduction aujourd hui. Elle concerne la forme. Au cours de la lecture, nous avons relevé plus de cinquante coquilles, au sens de "faute (orthographe ou autre) que le correcteur a laissé passer" (Ramat. Grammaire typographique), dont quelques "classiques": occurence (p. 39), Toute autre est la réaction (78), ils se sont rendus compte (185), Massachussets (187)... On note également. dans tout l'ouvrage, un emploi abusif des guillemets anglais, normalement réservés aux citations de deuxième rang. Plus gênantes sont les cocpuilles à l'intérieur des citations. Valery Larbaud, auteur d'une Lettre aux impriméurs qui demeure très actuelle. aurait sans aucun doute éprouvé beaucoup «de peines et d'angoisses" en découvrant pas moins de trois coquilles (dont l'infamante "presqu'imperceptiblement") dans les quelques lignes qui lui sont consacrées (36). Trois auteurs, mesdames Foz. Salama-Carr et Seleskovitch, constateront que le titre de leur ouvrage donné en référence comporte une faute d'orthographe (168, 222), tandis que 
deux autres, Hutchins et Somers, changent trois fois d'initiales en l'espace de quelques pages $(170,184,185)$ ! Il est vrai qu'à l'ère de la galaxie Gutenberg, du traitement de texte et de l'éditique, l'intervention traditionnelle des préparateurs de copie et des correcteurs d'épreuves semble souvent escamotée, les éditeurs se limitant à un simple rôle d'imprimeurs/diffuseurs. (Meta a déjà publié des comptes rendus signalant cette anomalie dans le cas de maisons d'édition se disant «érudites» ou «universitaires».) Les auteurs d'ouvrages ainsi maltraités (ou mal traités) se devraient de réagir, car, volens nolens, c'est leur crédibilité qui est en jeu, notamment lorsqu'il s'agit d'universitaires s'adressant à des spécialistes de la langue et de la communication.

Toujours au chapitre de la forme, l'ouvrage témoigne d'une volonté didactique qui se manifeste notamment par une langue claire, urésistant à la tentation de créer des néologismes" et autres hermétismes, ainsi que par la présentation typographique : numérotation décimale, nombreux intertitres, paragraphes courts.

D'une façon générale. le principal objectif visé - présenter une vue synthétique de la traduction aujourd'hui dans l'optique de la théorie interprétative - est atteint. Nous ne sommes pas convaincu, par contre, que l'ouvrage de Marianne Lederer convertira les sceptiques ni même gagnera de nombreux nouveaux adeptes à la cause du modèle interprétatif, tant est grand, selon nous, l'écart entre le principe fondamental maintes fois affirmé, de l'Avant-propos à la quatrième de couverture - «Traduire, c'est comprendre le sens d'un texte original et le restituer» - et son application sous forme d'exemples/modèles de traduction de l'anglais au français. Après tout, c'est d'après les fruits qu'on juge l'arbre. Et c'est regrettable, s'agissant d'une École qui, sans révolutionner la théorie ni la pratique de la traduction, a contribué au renouvellement de sa pédagogie, enrichi le débat sur l'éternelle question: «Comment faut-il traduire ?», et produit des diplômés qui lui font honneur.

Paul A. Horguel. Université de Montréal. Montréal. Canada 